\title{
REDACCIÓN DE CASOS CLÍNICOS
}

\section{CLINICAL CASE WRITING}

\section{Iván BARRIOS ${ }^{1}$, Tulio DUARTE².}

${ }^{1}$ Universidad Nacional de Asunción, Facultad de Ciencias Exactas y Naturales, San Lorenzo, Paraguay. ${ }^{2}$ Universidad Nacional de Asunción, Facultad de Ciencias Médicas, Santa Rosa del Aguaray, Paraguay.

Cómo citar este artículo: Barrios I, Duarte T. Redacción de Casos Clínicos. Med. clín. soc. 2019;3(1):3940.

\section{Estimado Editor,}

El motivo de esta carta es compartir con los lectores algunos puntos clave a tener en cuenta para redactar casos clínicos para facilitar su publicación en alguna revista de impacto. La publicación de un caso clínico sirve para divulgar la experiencia adquirida por sus autores con una persona con alguna enfermedad. Su fin es transmitir conocimientos y formular hipótesis a partir de observaciones particulares.

Diversos autores han planteado la relevancia de publicar casos clínicos, sin embargo, los que defienden su publicación mencionan la importancia de dar a conocer a la comunidad científica presentaciones clínicas poco habituales, tratamientos novedosos, complicaciones inesperadas, etc. (1).Haciendo énfasis pues a debe existir la premisa de la novedad y relevancia clínica, ya que no sirve de nada publicar la presentación y evolución esperadas de una enfermedad, puesto que se eso se puede consultar en fuentes primarias.

El caso elegido debe tener un diagnóstico conocido, plantear un dilema clínico y contener algún elemento nuevo, raro, importante o interesante. Puede tratarse de una enfermedad, un efecto adverso, un agente etiológico, un mecanismo fisiopatológico, una manifestación inesperada, una asociación sorprendente o un desenlace imprevisto. También sirven aspectos anatómicos o semiológicos inéditos, y nuevos usos de las modalidades diagnósticas (1).

Una vez que hemos redactado el caso clínico y sus anexos se debe redactar la introducción y la discusión. La introducción debe ser una invitación al lector para que continúe leyendo el artículo. Por ello debe ser breve. Debe informar de lo que se conoce del tema en forma sintética y dar los motivos para comunicar el caso. Cada afirmación obtenida de uno de los artículos revisados debe citarse (2). En la discusión se debe resaltar lo más importante, integrar los conocimientos revisados y lo aportado por el caso clínico. Se debe informar si se ha generado nuevo conocimiento y su utilidad en la práctica clínica (2).

Luego de haber escrito la introducción, el caso clínico y la discusión hay que seleccionar en qué revista es más pertinente publicar el caso clínico, al ser un artículo con especial relevancia en la práctica clínica hay que enfocarse en revistas especializadas en el campo de la investigación realizada, puesto que sus lectores son la mejor población diana o posible población interesada en leer sobre el caso clínico. 
Así mismo el resumen, y en algunos casos la conclusión, deben ser redactados teniendo en cuenta las normas brindadas por los editores de la revista en la que hay interés de publicar, todo esto también teniendo en cuenta el estilo de la bibliografía solicitado.

A nuestro criterio, más importante que la correcta redacción de un caso clínico, es preguntarse sobre su pertinencia, por lo que se vuelve a remarcar los puntos a tener en cuenta ante la pregunta ¿debo publicar este caso clínico?

Las razones más comunes para publicar un caso son las siguientes: 1) una asociación inesperada entre enfermedades o síntomas; 2 ) un evento inesperado en el curso de observación o tratamiento de un paciente; 3 ) hallazgos que arrojan nueva luz sobre la posible patogénesis de una enfermedad o un efecto adverso; 4) características únicas o raras de una enfermedad; 5) enfoques terapéuticos únicos; Variación de las estructuras anatómicas.

\section{CONFLICTOS DE INTERÉS Y FUENTE DE FINANCIACIÓN}

Los autores declaran no poseer conflictos de interés. Fuente de financiación: ninguna.

\section{REFERENCIAS BIBLIOGRÁFICAS}

1. Mellado JM, Packer CD. El caso clínico: vigencia, valores y estrategias para abordar su escritura. Radiología. 2017;59(6):496-503. https://doi.org/10.1016/j.rx.2017.07.002

2. Vega J. Cómo escribir y publicar un caso clínico: Guía práctica. Revista médica de Chile. 2015;143(4):499-505. https://doi.org/10.4067/S0034-98872015000400012

3. Guidelines to writing a clinical case report. Heart Views. 2017;18(3):104-105. https://dx.doi.org/10.4103\%2F1995-705X.217857 